\title{
Artificial intelligence for automatic cerebral ventricle segmentation and volume calculation: a clinical tool for the evaluation of pediatric hydrocephalus
}

\author{
*Jennifer L. Quon, MD, ${ }^{1}$ Michelle Han, MD, ${ }^{2}$ Lily H. Kim, MD, ${ }^{2}$ Mary Ellen Koran, MD, PhD, ${ }^{3}$ \\ Leo C. Chen, MD, ${ }^{4}$ Edward H. Lee, PhD, ${ }^{5}$ Jason Wright, MD, ${ }^{6}$ Vijay Ramaswamy, MD, PhD, \\ Robert M. Lober, MD, PhD, ${ }^{8}$ Michael D. Taylor, MD, PhD, ${ }^{9}$ Gerald A. Grant, MD, ${ }^{1,11}$ \\ Samuel H. Cheshier, MD, PhD, ${ }^{10}$ John R. W. Kestle, MD, ${ }^{10}$ Michael S. B. Edwards, MD, ${ }^{1,11}$ and \\ Kristen W. Yeom, MD 3,12
}

1Department of Neurosurgery, Stanford University School of Medicine; ${ }^{2}$ Stanford University School of Medicine; ${ }^{3}$ Department of Radiology, Stanford University School of Medicine; ${ }^{4}$ Department of Urology, Stanford University School of Medicine; ${ }^{5}$ Department of Electrical Engineering, Stanford University School of Engineering, Stanford, California; ${ }^{6}$ Department of Radiology, Seattle Children's Hospital, University of Washington School of Medicine, Seattle, Washington; Section of Neuro-Oncology, The Hospital for Sick Children, University of Toronto, Ontario, Canada; ' $D$ Department of Neurosurgery, Dayton Children's Hospital, Wright State University Boonshoft School of Medicine, Dayton, Ohio; 'Division of Neurosurgery, The Hospital for Sick Children, University of Toronto, Ontario, Canada; ${ }^{10}$ Department of Neurosurgery, University of Utah School of Medicine, Salt Lake City, Utah; and Divisions of ${ }^{11}$ Pediatric Neurosurgery and ${ }^{12}$ Pediatric Neuroradiology, Lucile Packard Children's Hospital Stanford, Stanford, California

OBJECTIVE Imaging evaluation of the cerebral ventricles is important for clinical decision-making in pediatric hydrocephalus. Although quantitative measurements of ventricular size, over time, can facilitate objective comparison, automated tools for calculating ventricular volume are not structured for clinical use. The authors aimed to develop a fully automated deep learning (DL) model for pediatric cerebral ventricle segmentation and volume calculation for widespread clinical implementation across multiple hospitals.

METHODS The study cohort consisted of 200 children with obstructive hydrocephalus from four pediatric hospitals, along with 199 controls. Manual ventricle segmentation and volume calculation values served as "ground truth" data. An encoder-decoder convolutional neural network architecture, in which T2-weighted MR images were used as input, automatically delineated the ventricles and output volumetric measurements. On a held-out test set, segmentation accuracy was assessed using the Dice similarity coefficient ( 0 to 1 ) and volume calculation was assessed using linear regression. Model generalizability was evaluated on an external MRI data set from a fifth hospital. The DL model performance was compared against FreeSurfer research segmentation software.

RESULTS Model segmentation performed with an overall Dice score of 0.901 ( 0.946 in hydrocephalus, 0.856 in controls). The model generalized to external MR images from a fifth pediatric hospital with a Dice score of 0.926 . The model was more accurate than FreeSurfer, with faster operating times (1.48 seconds per scan).

CONCLUSIONS The authors present a DL model for automatic ventricle segmentation and volume calculation that is more accurate and rapid than currently available methods. With near-immediate volumetric output and reliable performance across institutional scanner types, this model can be adapted to the real-time clinical evaluation of hydrocephalus and improve clinician workflow.

https://thejns.org/doi/abs/10.3171/2020.6.PEDS20251

KEYWORDS cerebral ventricles; ventricular volume; hydrocephalus; artificial intelligence; machine learning; deep learning 
$\mathrm{M}$ ORE than 100 years ago, in his seminal paper on pneumoencephalography, Walter Dandy reported the first technique for visualizing the cerebral ventricles. ${ }^{1}$ Since then, imaging of the ventricles on CT or MRI scanners has become routine in caring for patients with hydrocephalus and other CSF abnormalities. ${ }^{2}$ Ventricular access procedures, from placement of external ventricular drains to insertion of ventricular shunts, are some of the most common and critical neurosurgical interventions. ${ }^{3,4}$ Changes in ventricular size and shape can help clarify a patient's clinical status and thus guide neurosurgical decision-making. However, radiologists and neurosurgeons typically assess ventricular size by visual inspection alone. The evaluation of ventricular size over time is also complicated by differences in slice thickness, head angulation, and sequence type between different scans, which often limit direct comparison. Furthermore, linear estimates, such as the frontal-occipital horn ratio (FOR), can be used to assess ventricular size but do not fully capture changes in ventricular volume. ${ }^{5-7}$ Volume calculations, in turn, depend on segmentation of the cerebral ventricles, which is time-consuming and labor-intensive when manually performed. Nevertheless, consistently and accurately tracking ventricular volumes would significantly improve and standardize the lifelong clinical management of patients with hydrocephalus.

Limited automated tools for ventricle segmentation exist, but they are primarily research software that is not well suited for real-time clinical use. They require volumetric 3D T1-weighted scans with lengthier acquisition times, time-consuming image processing, and manual quality control. ${ }^{8-11}$ Additionally, most are tailored to healthy adult brains and do not have high fidelity in pediatric brains or in brains with distortion, such as from an underlying tumor. Automated methods for evaluating pediatric brain substructures have also been limited to small cohorts. ${ }^{8,12,13}$

To address the clinical need for an efficient, reliable, and quantitative method to evaluate the cerebral ventricles, we developed a deep learning (DL)-based clinical tool to consistently evaluate ventricular volume, over time, for patients with hydrocephalus.

\section{Methods}

\section{Cohort Selection and Study Design}

After institutional review board approval from each institution, data use agreements were developed between the host institution (Lucile Packard Children's Hospital, Stanford University) and four pediatric hospitals for this multicenter study. A retrospective review of brain MRI obtained between 2002 and 2018 was used to identify patients $\leq 22$ years of age with posterior fossa (PF) brain tumors causing radiographically confirmed ventriculomegaly (Fig. 1). Hydrocephalus may be difficult to diagnose in the absence of symptoms or enlarged ventricles. Therefore, for the purposes of this study we targeted patients who presented acutely with obstructive hydrocephalus. Specifically, we chose patients with PF tumors, because they represent a more homogeneous cohort presenting with an obstructive etiology requiring intervention. The primary hydrocephalus cohort comprised 200 patients with PF tumors causing obstructive hydrocephalus (host institution, $n=50$; Seattle Children's Hospital, $n=75$; The Hospital for Sick Children, $\mathrm{n}=60$; and Dayton Children's Hospital, $n=15$ ). All patients were clinically symptomatic (headache, nausea, or vomiting) with MRI obtained prior to tumor treatment. We included patients who underwent emergency external ventricular drain placement, but excluded patients who had undergone permanent shunting, third ventriculostomy, or tumor debulking prior to their first available MRI sequences. From our database of neurologically normal children with negative MRI, 200 scans from 199 control patients were selected using random stratified sampling by age. ${ }^{14}$ All patients had T2-weighted scans. In all controls and a subset of the hydrocephalus patients, 3D T1-weighted spoiled gradient-recalled acquisition (SPGR), magnetization-prepared rapid acquisition gradient echo (MPRAGE), or 3D volume (BRAVO) T1weighted images were obtained. A board-certified neuroradiologist (K.W.Y.; more than 11 years of experience) performed image quality control.

The study cohort (400 T2-weighted scans) was divided into training $(\mathrm{n}=266 ; 137$ controls and 129 hydrocephalus) and validation ( $n=67 ; 34$ controls and 33 hydrocephalus) sets for model development and optimization, and a held-out test set ( $\mathrm{n}=67 ; 29$ controls, 38 hydrocephalus) for final evaluation of model performance. To further evaluate model generalizability in the clinical realm, performance was prospectively tested on T2-weighted scans obtained in patients with hydrocephalus from a fifth institution (Utah Primary Children's Hospital, $\mathrm{n}=9$ ). Scans from this independent institution were not used for model development and therefore served as a proxy for model performance in the context of clinical use outside of the original institutions.

\section{MRI Acquisition}

Across the five institutions, MRI was performed at either $1.5 \mathrm{~T}$ or $3 \mathrm{~T}$ using the following magnets: GE (GE Healthcare); Siemens (Siemens AG); Philips (Philips Healthcare); and Toshiba (Canon Medical Systems USA, Inc.). The T2-weighted scans were as follows: T2 turbo spin echo clear/sense, T2 fast spin echo, T2 propeller, T2 blade, T2 drive sense (TR/TE 2475.6-9622.24 msec/80$146.048 \mathrm{msec}$ ); slice thickness $1-5 \mathrm{~mm}$ with 0.5 - or $1-\mathrm{mm}$ skip; and matrix ranges of (224-1024) × (256-1024). The T1-weighted scan protocol comprised T1-weighted SPGR, BRAVO, or MPRAGE sequences; TR/TE 6.94-8.536 $\mathrm{msec} / 3.02-3.824 \mathrm{msec}$; slice thickness $0.8-1.2 \mathrm{~mm}$; and matrix $(256-512) \times(256-512)$.

\section{Ground Truth Labels}

Manual segmentation of the cerebral ventricles was performed on all axial slices by a neurosurgery resident (J.L.Q.) and a medical student (L.H.K.). Segmentations were reviewed by a board-certified pediatric neuroradiologist (K.W.Y.). Ventricle segmentations began superiorly at the first axial slice that contained the visible lateral ventricles and continued inferiorly to the foramina of Magendie and Luschka. The lateral, third, and fourth ventricles were 


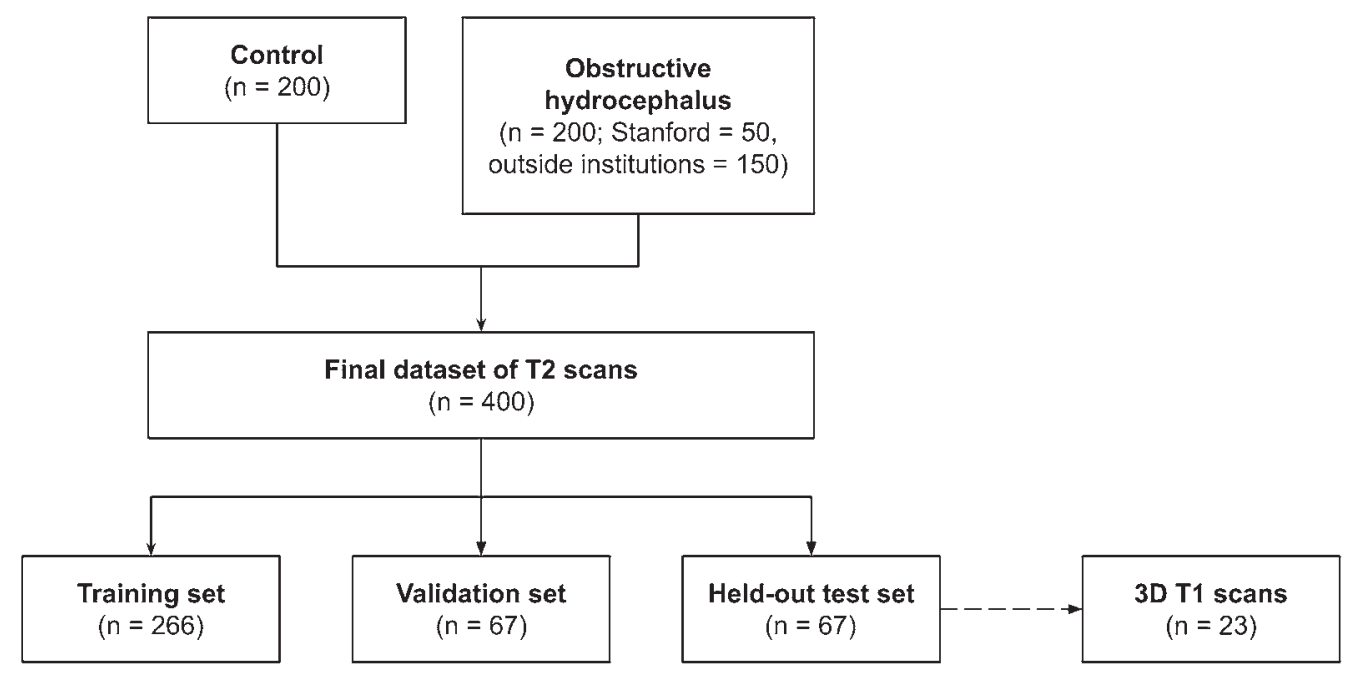

FIG. 1. Flow diagram demonstrating patient selection.

segmented, including the choroid plexus. When visible, the subarachnoid cisterns and cavum pellucidum were excluded. For the hydrocephalus cohort, the visible ventricular system (including the fourth ventricle) not obliterated by the PF tumor was segmented. Manual segmentations were performed using ITK-SNAP software. ${ }^{15}$

\section{Image Processing and Data Augmentation}

Axial DICOM images were preprocessed using the Python programming language. Skull stripping was not performed. Axial slices were resampled to $256 \times 256$ pixels. Data augmentation was performed by incorporating random rotations and flips.

\section{Model Architecture and Training}

We adapted a 2D U-net, ${ }^{16}$ a state-of-the-art convolutional neural network (CNN) architecture, to automatically segment the cerebral ventricles using $\mathrm{T} 2$-weighted scans as input. Our model consisted of an encoder-decoder structure: an encoder maps a volume to an abstract lowresolution encoding, and a decoder expands this encoding back to a full-resolution segmentation image, which specifies the probability that each pixel in a slice is ventricle or not ventricle (Supplementary Fig. 1). The encoder weights were adapted from a visual geometry group (VGG-11) architecture pretrained on the ImageNet data set, a consortium of more than 1.2 million 2D images. ${ }^{17}$ The model was trained to minimize the Dice $\operatorname{loss}^{18}$ of the segmentation output. Training was carried out on four Titan Xp graphics processing units (NVIDIA Corp.).

\section{FreeSurfer}

After visual inspection of 3D T1-weighted scans to parcellate the brain into tissue classes and derive quantitative estimates of volume, an automated, nonbiased, atlas-based Bayesian segmentation procedure was applied in FreeSurfer (FS) version 5.0 (http://surfer.nmr.mgh.harvard. edu/)..$^{19}$ FS preprocessing was performed using a previ- ously published methodology. ${ }^{19,20}$ Quantitative estimates of volume were derived for spatially distinct regions of interest, as specified in the Desikan-Killiany atlas..$^{21} \mathrm{FS}$ was run using a central processing unit due to the lack of active support for graphics processing unit use.

\section{Ventricular Volume Calculation}

"Ground truth" volumes were derived from manual segmentations of the ventricles, whereas the model-predicted ventricular volume was calculated using the predicted ventricle segmentation mask. Ventricular volume was calculated by multiplying the segmentation area of each axial slice by the slice thickness, and then summing the contribution from all slices. Volume calculations included "skip" in scans of noncontiguous slices. For FS, ventricular volume was automatically calculated by the software using the FS-derived ventricle segmentation mask.

\section{FOR and Evans' Ratio}

Linear measurements of the ventricles and skull included the FOR and Evans' ratio. ${ }^{5}$ Both measurements were manually determined on T2-weighted scans by using the measurement tool on ITK-SNAP software..$^{15}$

\section{Statistical Analyses}

Predicted ventricle segmentations were compared with ground truth data by using the Dice similarity coefficient $(2 \times$ true positives/ $[2 \times$ true positives + false positives + false negatives])..$^{18}$ Linear regression was used to evaluate volume calculations derived from T2-weighted scans versus 3D T1-weighted scans, as well as predicted versus ground truth segmentations. ANOVA was performed using multiple regression analysis of the Dice score, with the cohort group, ventricular volume, and age as independent variables. Dice coefficients from FS and the DL model were compared using a paired two-tailed t-test. Statistics calculations were performed using R version 3.5.1 (RStudio). 

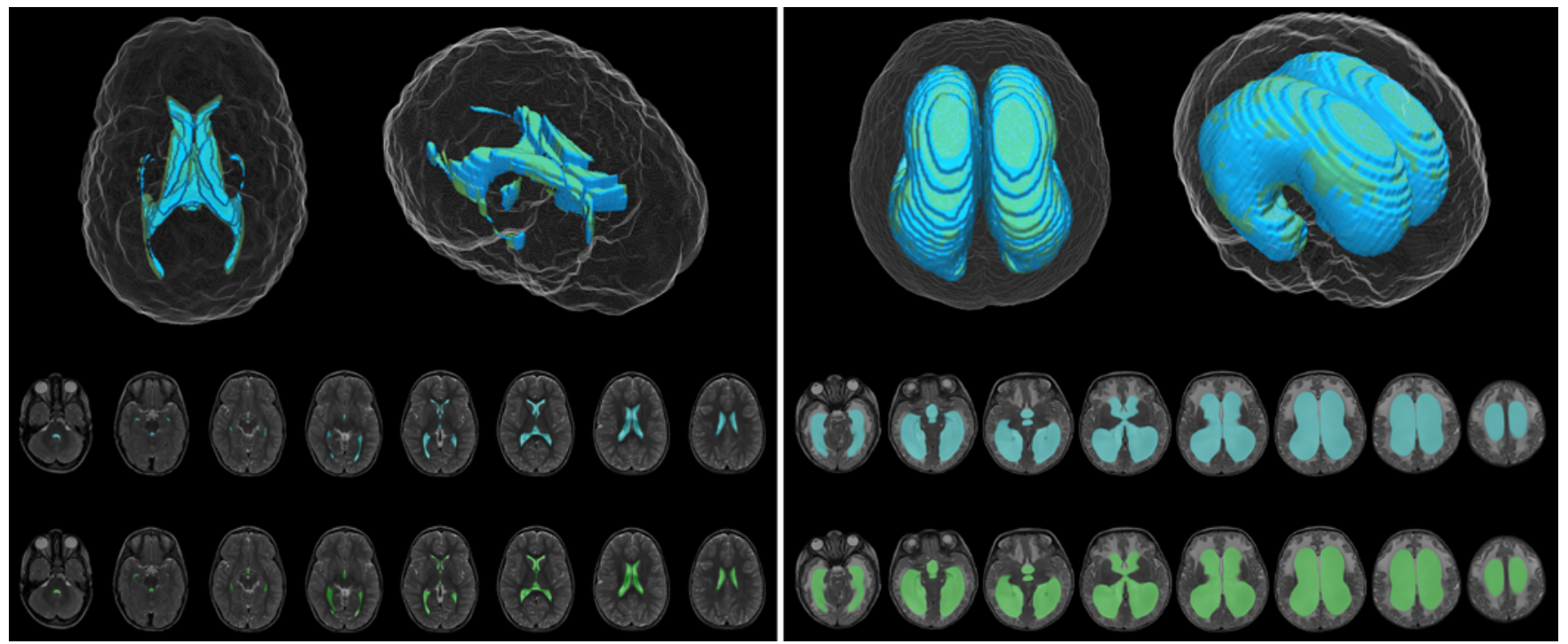

FIG. 2. DL model (blue) and ground truth manual (green) segmentation of representative control (left) and hydrocephalus (right) T2-weighted MR images.

\section{Results \\ Study Cohort}

The control cohort (199 patients, 200 scans) ranged from 0 to 19 years of age (median 8 years), with $48.7 \%$ $(\mathrm{n}=97)$ males. We visualized the ventricular volumes across ages to get an approximation of a normal population curve. In our cohort, dramatic increases in volume occurred during the first 50 months of life, with a plateau between 4 and 8 years, after which volume increased until approximately 14 years of age (Supplementary Fig. 2).

The obstructive hydrocephalus cohort $(\mathrm{n}=200)$ ranged from 0 to 22 years of age (median 5 years) with $56 \%$ (n $=112$ ) males. Twenty-seven patients with hydrocephalus had emergency external ventricular drain placement prior to MRI due to clinical instability but continued to have imaging findings of hydrocephalus.

\section{Calculation of Ventricular Volume on 3D T1-Weighted Scans Is Comparable to That of T2-Weighted Scans}

Three-dimensional T1-weighted scans, with thinner slices and greater anatomical detail, have commonly been chosen for volumetric analysis. Given that T2-weighted scans have wider clinical use, we demonstrate that ventricular volumes manually determined using T2-weighted scans are comparable to manual calculations based on 3D T1-weighted scans (Supplementary Fig. 3). Despite the larger and more variable slice thickness for T2-weighted scans $(2-6 \mathrm{~mm})$ compared with 3D T1-weighted scans (1 $\mathrm{mm}$ ), volume calculations derived from the two sequences correlated closely $\left(r^{2}=0.76\right)$.

\section{Model Performance}

On a held-out test set of T2-weighted scans, model segmentation was very accurate, with an overall Dice score of 0.901. Segmentation and volume calculation were more accurate in patients with hydrocephalus (Dice coefficient 0.946) than in control patients (Dice coefficient 0.856$)\left(\mathrm{F}=375.4, \mathrm{p}<2.2 \times 10^{-16}\right)$ (Figs. 2 and 3, Videos 1 and 2).

VIDEO 1. 3D reconstruction of DL model (blue) and ground truth manual (green) segmentation of a representative control T2-weighted MR image. Copyright Kristen W. Yeom. Published with permission. Click here to view.

VIDEO 2. 3D reconstruction of DL model (blue) and ground truth manual (green) segmentation of a representative hydrocephalus T2-weighted MR image. Copyright Kristen W. Yeom. Published with permission. Click here to view.

Within each group, model performance was better in patients with higher ventricular volumes (control: $r=0.39, p=$ $7.3 \times 10^{-9}$; hydrocephalus: $\left.\mathrm{r}=0.59, \mathrm{p}<2.2 \times 10^{-16}\right)$. Segmentation accuracy did not vary with age $(\mathrm{F}=1.39, \mathrm{p}=0.24)$. The model's volume calculation correlated strongly with the FOR and Evans' ratio $\left(r^{2}=0.92\right.$ and 0.79 , respectively).

To further test the generalizability of our model, we evaluated segmentation accuracy on 9 patients with hydrocephalus from a fifth institution that was not used for initial model development. Accuracy was maintained even on this completely external test set (Dice coefficient 0.926 ; Fig. 3). Model evaluation took 1.48 seconds per scan at the graphics processing unit level (projected to 25 minutes at the central processing unit level).

\section{Comparison to FS}

FS was used for ventricle segmentation and volume calculation on a subset of the held-out test set with available 3D T1-weighted scans. ${ }^{22}$ Given that only 12 hydrocephalus patients from the held-out test set had 3D T1-weighted scans, we selected 11 age-matched controls to evaluate with FS. Ages ranged from several months to 13 years for both groups (median 7 years in controls, 6.5 years in hydrocephalus patients). FS was only able to segment 8 of 11 control group and 9 of 12 hydrocephalus group 3D T1weighted scans, with processing errors generated on the 

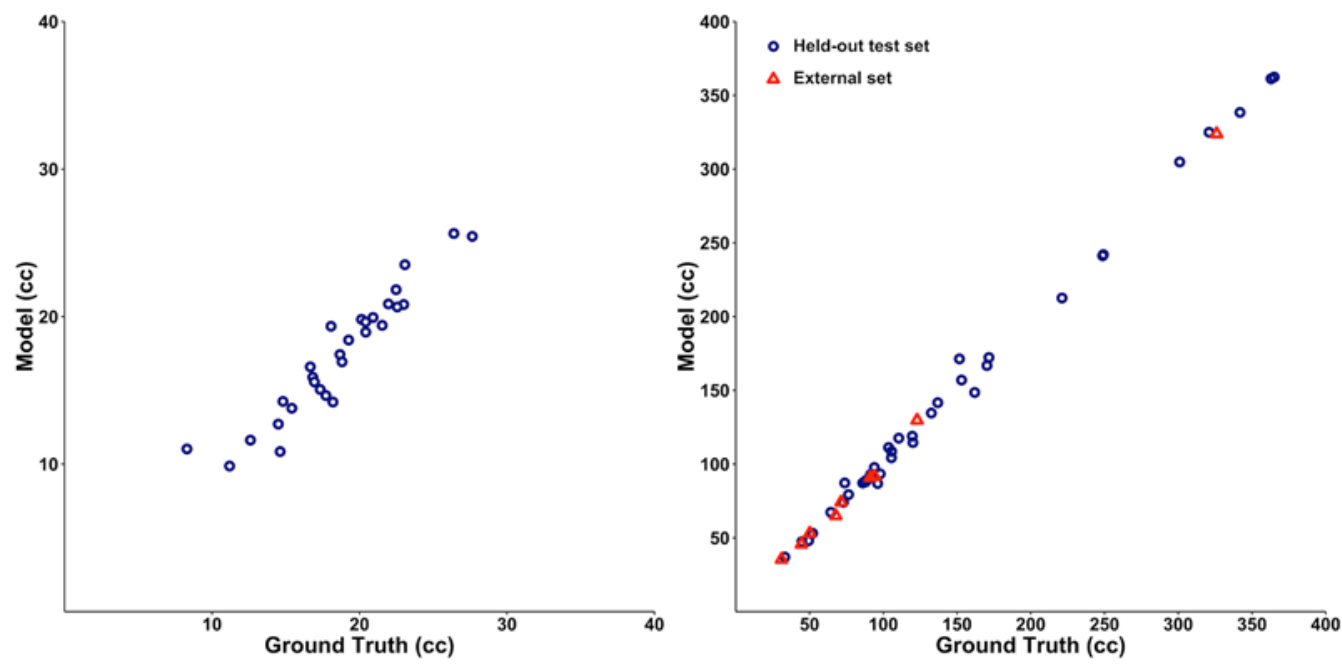

FIG. 3. Correlation between the DL model and manually derived ventricular volume for control (left) $\left(r^{2}=0.90\right)$ and hydrocephalus (right) $\left(r^{2}=0.997\right)$ T2-weighted MR images from the held-out test set. Blue circles denote MR images from the held-out test set. Red triangles denote external MR images from the fifth institution, not used for model development. Figure is available in color online only.

remaining scans. Of scans that FS successfully processed, the Dice coefficients were 0.55 for control and 0.86 for hydrocephalus 3D T1-weighted scans (Figs. 4 and 5). The DL model was more accurate for the exact same patients (Dice coefficients of 0.83 for controls $\left[p=6.4 \times 10^{-5}\right.$ ] and 0.94 for hydrocephalus $\left.\left[\mathrm{p}=8.5 \times 10^{-5}\right]\right)$. Per patient scan, FS took 8.2 to 207.3 hours (median 20.3 hours) for ventricle segmentation and volume output, compared with 1.48 seconds per patient scan for the DL model.

\section{Discussion}

In this study, we present a DL model to calculate ventricular volume for pediatric patients with and those with- out obstructive hydrocephalus by using T2-weighted MR images. We trained and tested a state-of-the-art DL architecture on a large cohort of 400 scans from four different hospitals, representing the largest study of its kind to date. Our heterogeneous imaging data set consisted of scans from geographically distinct institutions as well as from multiple scanner vendors and at different magnet strengths. To simulate clinical implementation and evaluate model performance at an entirely new venue, we prospectively applied our model to a fifth institution that made no contributions to model development. Our model could therefore be reliably implemented across MR scanners without degradation of its performance.
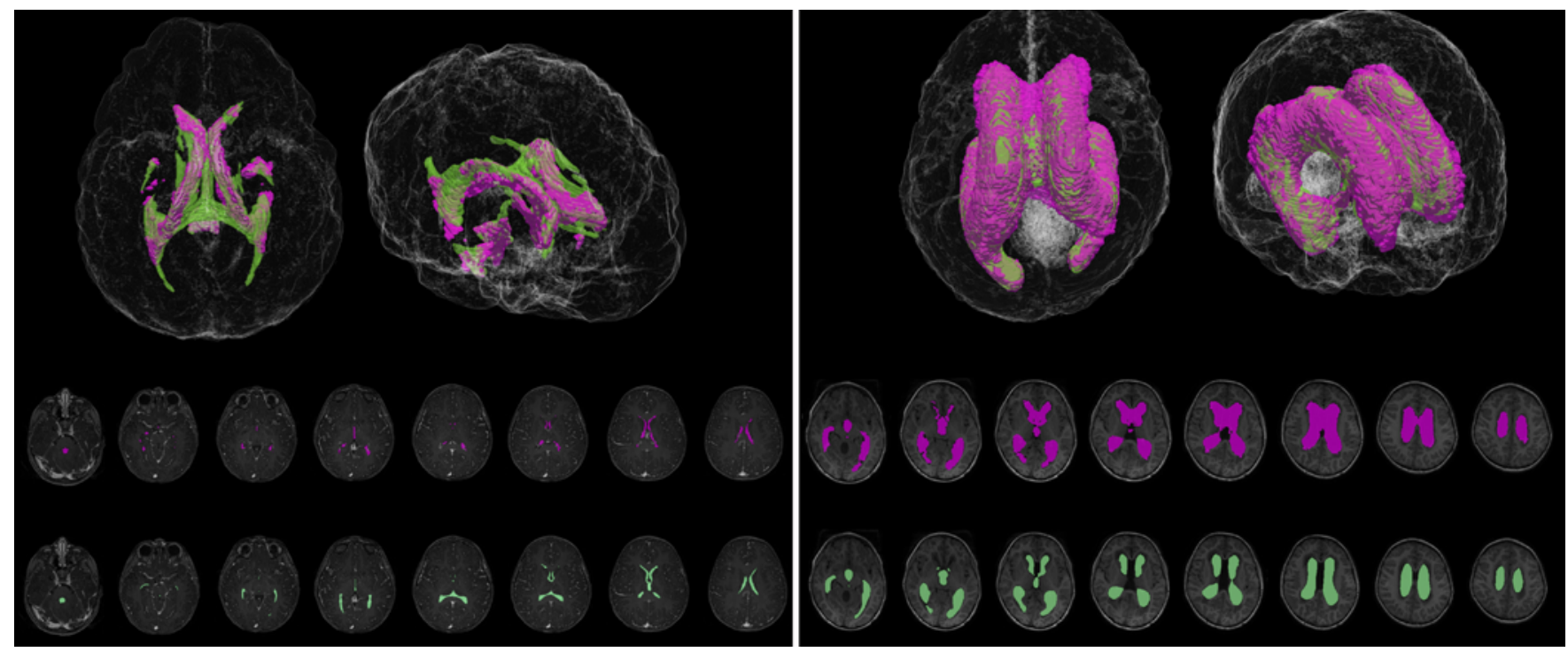

FIG. 4. FS (purple) and ground truth manual (green) segmentation of representative control (left) and hydrocephalus (right) 3D T1-weighted MR images. 

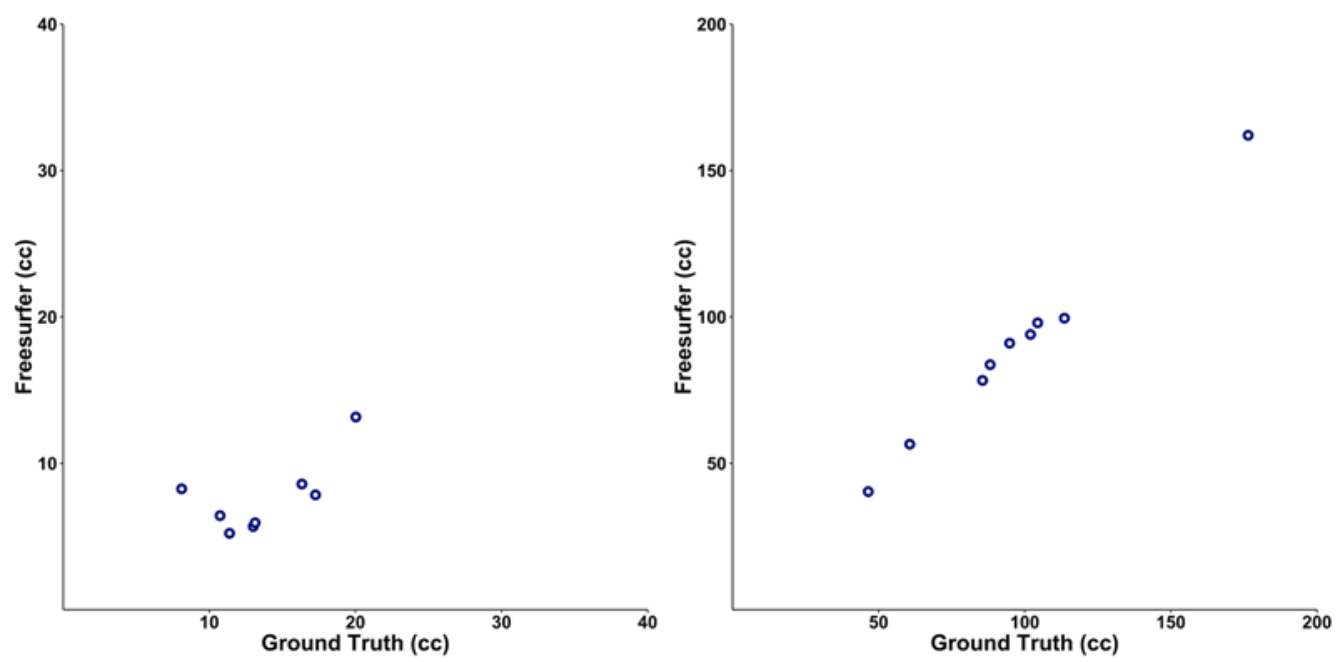

FIG. 5. Correlation between the FS and manually derived ventricular volume for a subcohort of control (left) $\left(r^{2}=0.44\right)$ and hydrocephalus (right) $\left(r^{2}=0.994\right) 3 D$ T1-weighted MR images. Figure is available in color online only.

Pediatric hydrocephalus has a prevalence of approximately 6 in 10,000 live births, and up to half of patients who undergo shunt insertion experience shunt malfunction within the first year of placement. ${ }^{23,24}$ Children with shunt-treated hydrocephalus therefore undergo numerous imaging studies for routine surveillance and clinical evaluation, but these are not always directly comparable, depending on the slice thickness, head angulation or rotation, and sequence type of each scan. For patients with shunts, ventricular monitoring would undoubtedly benefit from an objective, consistent, quantitative method for gauging ventricular volume change or stability over time. ${ }^{25}$ Yet, manual ventricle segmentation is cumbersome, and current automated methods are time-consuming and require significant oversight..$^{8-10,26-28}$ These methods have included explicit handcrafted feature extraction to segment specific tissue types, with additional human input for regions of interest, coregistration of reference atlases, thresholding, or further parameter modifications. ${ }^{8-10,26-28}$ Other research segmentation tools, including FS, have been applied mostly to small cohorts of healthy adult volunteers and have not been validated for use in children. ${ }^{7,22,27-31}$ Additionally, these approaches have relied on volumetric 3D T1-weighted scans, which take more time to acquire and are not routinely collected in the clinical setting.

Although there have been reports of pediatric brain segmentation, particularly in neonates, these studies have also been limited to small cohorts of healthy subjects. ${ }^{8,12,26,32}$ Most have also required significant manual input, relied on predetermined intensity and spatial information, and used tissue probability mapping or atlasbased approaches. ${ }^{8,12,13,26,32}$ These methods limit their applicability across institutions, where variations in magnet field strength and imaging protocols can create bias in volume measurements. ${ }^{33}$ Their fidelity is also largely unknown in cases in which brain pathology causes significant deformation and limits spatial registration, such as in patients with tumors or ventricular shunts. Additionally, changes in $\mathrm{T} 1$ and $\mathrm{T} 2$ signals that accompany brain de- velopment and myelination from infancy to adolescence ${ }^{31}$ have posed unique challenges to existing segmentation methods, ${ }^{8,34}$ which have not been developed using pediatric patients of all ages.

In contrast, by using DL, which does not depend on extraction of hand-crafted features, our model requires minimal preprocessing and human oversight after training is complete. Although one prior study has demonstrated the feasibility of DL for classifying brain tissue subtypes in a small cohort of normal infants, ${ }^{35}$ our data set is much larger and consisted of scans from infants, children, and adolescents, some with and some without hydrocephalus. Despite age-related white matter T2 signal changes, our model's accuracy did not vary with age, suggesting that the DL model is agnostic to myelination status. Importantly, head angulation and rotation, often an issue for neonates and smaller children in the scanner, did not degrade the ability to measure ventricular volume. Despite the presence of ventricular catheters in 27 patients, the DL model still reliably calculated ventricular volume, suggesting that it would be able to do so even after permanent ventricular shunt placement.

Interestingly, our model showed better performance in the hydrocephalus cohort compared with controls. Given that segmentation is a pixel-based classification task, this is probably due to the higher ratio of ventricle to brain pixels associated with larger ventricular volumes. Therefore, larger ventricles in the setting of PF tumors provided more pixel-level training material for the DL model to learn the ventricle segmentation task. In addition, small errors made by the model may have had a larger negative impact on its performance score in the context of smaller ventricles.

Our model has several limitations. Because our training data did not distinguish between the different ventricular compartments, our model does not identify compartment-specific volumes, which would be useful in cases of ventricular entrapment. Trapped cystic pockets or other CSF abnormalities such as porencephalic and arachnoid 
cysts would not necessarily be parcellated from the remainder of the ventricular system. We also did not train our model to determine the presence or absence of hydrocephalus, because this determination often requires additional clinical correlation. Therefore, this technique would still need to be associated with the clinical status of the patient in order to make the best decisions. In addition, our training data only included children with normal ventricular anatomy and those with symptomatic obstructive hydrocephalus from PF tumors causing obvious ventriculomegaly.

Our goal in this initial study was to select a cohort of patients with hydrocephalus that was as homogeneous as possible in terms of their etiology and presentation. Nevertheless, we recognize that many etiologies and manifestations of hydrocephalus exist, such as normal pressure hydrocephalus, hydrocephalus ex vacuo, slit ventricles, aqueductal stenosis, and communicating hydrocephalus. Thus, although our model would probably be able to calculate ventricular volume in these cases, its performance accuracy needs to be validated in the context of these other etiologies. Future directions involve using the model to prospectively evaluate ventricular volume in the context of more heterogeneous etiologies of hydrocephalus, as well as in the setting of suspected shunt malfunction. In particular, determining whether the model is able to detect small changes in CSF volume would indicate its applicability to some of the most challenging clinical scenarios, such as subtle or seemingly "radiographically occult" shunt malfunctions.

Notably, the model was trained to include the visible fourth ventricle volume by using images from the control cohort. In cases of PF tumors obliterating the fourth ventricle, the model was trained to include the volume of the visible fourth ventricle, and to exclude tumor tissue from the ventricular volume calculation. Although fourth ventricle volume would probably be included in the overall ventricular volume calculation of other hydrocephalus etiologies, performance would again need to be validated in those cohorts. Our DL model nonetheless lays the groundwork for more widespread applications and could be used to pretrain future model iterations. Future studies could also examine model performance by using other T2 derivatives, such as single-shot spin echo or various $\mathrm{T} 2$ weighted imaging used for fast MRI, for even more widespread applicability.

Despite its limitations, our model quickly and accurately segments the ventricles in commonly acquired T2weighted scans, across a wide range of ventricular sizes, regardless of differences in myelination, degrees of brain deformation, or the presence of periventricular edema. Questions often arise regarding generalizability of artificial intelligence models derived from a single institution. In this study, we aimed to create a generalizable model using data from various hospitals and geographic regions that use different MR scanner vendors, magnet strengths, and imaging parameters. Although our results from a small external data set suggest model generalizability, more extensive prospective investigations are nevertheless needed to further validate model performance across global centers.

\section{Conclusions}

We developed an artificial intelligence-based clinical tool to automatically segment the cerebral ventricles and calculate ventricular volume such that ventricular volume can be consistently measured in pediatric patients with obstructive hydrocephalus. Clinical deployment would facilitate neurosurgical decision-making and be reliable across different institutional scanners. Although its use in various clinical scenarios still requires correlation with symptomatology, and even brain volume, our model automatically provides useful volumetric data for the ventricles. Future directions involve evaluating model performance in the context of other hydrocephalus etiologies and CSF compartments, as well as further evaluation of prospective performance.

\section{References}

1. Dandy WE. Röntgenographyof the brain after the injection of air into the spinal canal. Ann Surg. 1919;70(4):397-403.

2. McInerny TK, Adam HM, Campbell DE, et al. AAP Textbook of Pediatric Care. American Academy of Pediatrics; 2016.

3. Patwardhan RV, Nanda A. Implanted ventricular shunts in the United States: the billion-dollar-a-year cost of hydrocephalus treatment. Neurosurgery. 2005;56(1):139-145.

4. Pereira S, Pinto A, Oliveira J, et al. Automatic brain tissue segmentation in MR images using random forests and conditional random fields. J Neurosci Methods. 2016;270:111-123.

5. O'Hayon BB, Drake JM, Ossip MG, et al. Frontal and occipital horn ratio: a linear estimate of ventricular size for multiple imaging modalities in pediatric hydrocephalus. Pediatr Neurosurg. 1998;29(5):245-249.

6. Kulkarni AV, Drake JM, Armstrong DC, Dirks PB. Imaging correlates of successful endoscopic third ventriculostomy. $J$ Neurosurg. 2000;92(6):915-919.

7. Multani JS, Oermann EK, Titano J, et al. Quantitative computed tomography ventriculography for assessment and monitoring of hydrocephalus: a pilot study and description of method in subarachnoid hemorrhage. World Neurosurg. 2017; 104:136-141.

8. Wang L, Gao Y, Shi F, et al. LINKS: learning-based multisource integration framework for segmentation of infant brain images. Neuroimage. 2015;108:160-172.

9. Adams CM, Wilson TD. Virtual cerebral ventricular system: an MR-based three-dimensional computer model. Anat Sci Educ. 2011;4(6):340-347.

10. Ishii K, Soma T, Shimada K, et al. Automatic volumetry of the cerebrospinal fluid space in idiopathic normal pressure hydrocephalus. Dement Geriatr Cogn Disord Extra. 2013; 3(1):489-496.

11. Schnack HG, Hulshoff Pol HE, Baaré WFC, et al. Automatic segmentation of the ventricular system from MR images of the human brain. Neuroimage. 2001;14(1 Pt 1):95-104.

12. Anbeek P, Išgum I, van Kooij BJ, et al. Automatic segmentation of eight tissue classes in neonatal brain MRI. PLoS One. 2013;8(12):e81895.

13. Qiu W, Yuan J, Rajchl M, et al. 3D MR ventricle segmentation in pre-term infants with post-hemorrhagic ventricle dilatation (PHVD) using multi-phase geodesic level-sets. Neuroimage. 2015;118:13-25.

14. Forkert ND, Li MD, Lober RM, Yeom KW. Gray Matter Growth Is Accompanied by Increasing Blood Flow and Decreasing Apparent Diffusion Coefficient during Childhood. AJNR Am J Neuroradiol. 2016;37(9):1738-1744.

15. Yushkevich PA, Piven J, Hazlett HC, et al. User-guided 3D active contour segmentation of anatomical structures: significantly improved efficiency and reliability. Neuroimage. 2006; 31(3):1116-1128. 
16. Ronneberger O, Fischer P, Brox T. U-net: Convolutional networks for biomedical image segmentation. In: Navab N, Hornegger J, Wells W, Frangi A, eds: Medical Image Computing and Computer-Assisted Intervention-MICCAI 2015. Lecture Notes in Computer Science. Vol 9351. Springer; 2015:234-241. Accessed July 23, 2020. https://link.springer. com/chapter/10.1007\%2F978-3-319-24574-4_28

17. Deng J, Dong W, Socher R, et al. ImageNet: A large-scale hierarchical image database. Paper presented at: 2009 IEEE conference on computer vision and pattern recognition 2009. In: 2009 IEEE Conference on Computer Vision and Pattern Recognition. IEEE; 2009:248-255. Accessed July 23, 2020. https://ieeexplore.ieee.org/document/5206848

18. Dice LR. Measures of the amount of ecologic association between species. Ecology. 1945;26(3):297-302.

19. Dale AM, Fischl B, Sereno MI. Cortical surface-based analysis. I. Segmentation and surface reconstruction. Neuroimage. 1999;9(2):179-194.

20. Ségonne F, Pacheco J, Fischl B. Geometrically accurate topology-correction of cortical surfaces using nonseparating loops. IEEE Trans Med Imaging. 2007;26(4):518-529.

21. Desikan RS, Ségonne F, Fischl B, et al. An automated labeling system for subdividing the human cerebral cortex on MRI scans into gyral based regions of interest. Neuroimage. 2006;31(3):968-980.

22. Fischl B, Salat DH, Busa E, et al. Whole brain segmentation: automated labeling of neuroanatomical structures in the human brain. Neuron. 2002;33(3):341-355.

23. DeFlorio RM, Shah CC. Techniques that decrease or eliminate ionizing radiation for evaluation of ventricular shunts in children with hydrocephalus. Semin Ultrasound CT MR. 2014;35(4):365-373.

24. Wright Z, Larrew TW, Eskandari R. Pediatric hydrocephalus: current state of diagnosis and treatment. Pediatr Rev. 2016; 37(11):478-490.

25. Drake JM. The surgical management of pediatric hydrocephalus. Neurosurgery. 2008;62(Suppl 2):630-642.

26. Ourselin S, Styner MA, Moeskops P, et al. Evaluation of an automatic brain segmentation method developed for neonates on adult MR brain images. Proc SPIE. 2015;9413:941315.

27. Lemieux L, Hammers A, Mackinnon T, Liu RS. Automatic segmentation of the brain and intracranial cerebrospinal fluid in T1-weighted volume MRI scans of the head, and its application to serial cerebral and intracranial volumetry. Magn Reson Med. 2003;49(5):872-884.

28. Chen H, Dou Q, Yu L, et al. VoxResNet: Deep voxelwise residual networks for brain segmentation from 3D MR images. Neuroimage. 2018;170:446-455.

29. González-Villà S, Oliver A, Valverde S, et al. A review on brain structures segmentation in magnetic resonance imaging. Artif Intell Med. 2016;73:45-69.

30. Holden M, Schnabel JA, Hill DL. Quantification of small cerebral ventricular volume changes in treated growth hormone patients using nonrigid registration. IEEE Trans Med Imaging. 2002;21(10):1292-1301.

31. Moeskops P, de Bresser J, Kuijf HJ, et al. Evaluation of a deep learning approach for the segmentation of brain tissues and white matter hyperintensities of presumed vascular origin in MRI. Neuroimage Clin. 2017;17:251-262.

32. Beare RJ, Chen J, Kelly CE, et al. Neonatal brain tissue classification with morphological adaptation and unified segmentation. Front Neuroinform. 2016;10:12.
33. Jovicich J, Czanner S, Han X, et al. MRI-derived measurements of human subcortical, ventricular and intracranial brain volumes: Reliability effects of scan sessions, acquisition sequences, data analyses, scanner upgrade, scanner vendors and field strengths. Neuroimage. 2009;46(1):177-192.

34. McGraw P, Liang L, Provenzale JM. Evaluation of normal age-related changes in anisotropy during infancy and childhood as shown by diffusion tensor imaging. AJR Am J Roentgenol. 2002;179(6):1515-1522.

35. Zhang W, Li R, Deng H, et al. Deep convolutional neural networks for multi-modality isointense infant brain image segmentation. Neuroimage. 2015;108:214-224.

\section{Disclosures}

The authors report no conflict of interest concerning the materials or methods used in this study or the findings specified in this paper.

\section{Author Contributions}

Conception and design: Yeom, Quon, Han, Kim, Cheshier, Kestle, Edwards. Acquisition of data: Yeom, Quon, Han, Kim, Koran, Lee, Wright, Ramaswamy, Lober, Taylor, Grant. Analysis and interpretation of data: Yeom, Quon, Han, Kim, Chen, Lee, Edwards. Drafting the article: Quon, Han, Kim, Chen. Critically revising the article: Yeom, Quon, Han, Kim, Koran, Chen, Lober, Cheshier, Edwards. Reviewed submitted version of manuscript: all authors. Approved the final version of the manuscript on behalf of all authors: Yeom. Statistical analysis: Quon, Han, Kim, Koran, Chen. Administrative/technical/material support: Yeom, Quon, Edwards. Study supervision: Yeom, Quon.

\section{Supplemental Information Videos}

Video 1. https://vimeo.com/434085861.

Video 2. https://vimeo.com/434085879.

\section{Online-Only Content}

Supplemental material is available with the online version of the article.

Supplementary Figs. 1-3. https://thejns.org/doi/suppl/10.3171/ 2020.6.PEDS20251.

\section{Previous Presentations}

Preliminary findings from this work were presented as an oral presentation at the 2019 American Association of Neurological Surgeons (AANS) annual scientific meeting, held in San Diego, CA, and as a poster at the 2019 American Academy of Neurology (AAN) annual scientific meeting, held in Philadelphia, PA.

\section{Correspondence}

Kristen W. Yeom: Lucile Packard Children's Hospital, Stanford University School of Medicine, Palo Alto, CA. kyeom@ stanford.edu. 